\title{
THEORY OF DWARF NOVAE
}

\author{
G. T. B A T H \\ Dept. of Astrophysics, Oxford University, Oxford, England
}

\author{
If a star were confin'd into a Tomb \\ Her captive flames must needs burn there; \\ But when the hand that lockt her up, gives room, \\ She'l shine through all the sphere. \\ Henry Vaughan: Silex Scintillans, 1655.
}

\begin{abstract}
Recent work on the physical processes resulting from mass transfer between the red and blue components of dwarf nova binaries is reviewed. The optical behaviour of the blue component's accretion disc suggests that it may be the infall, accretion energy which is being liberated during outbursts. Theoretical results which suggest that the red component may suffer quasi-periodic mass transfer instabilities are discussed. The resulting accretion disc properties are considered and compared with the observed optical outburst behaviour for the simplest steady state disc models. The complexity of the interaction between the two stellar components in these systems is emphasized.
\end{abstract}

\section{The Problem}

The eruptive outbursts which characterise dwarf novae show a wide variety of behaviour. As Faulkner (1974) has pointed out, no simple unqualified statement about these objects is likely to be universally true, especially if one hopes eventually to account for the noneruptive behaviour of the nova-like systems, and the totally different behaviour of the classical nova - both of which seem to have the same underlying binary structure. Nonetheless, the variety of behaviour exhibited by dwarf novae should not be interpreted as meaning that one cannot isolate the physical processes that may be occurring in them. It is these physical processes that will be emphasised in this paper. The reader is referred to the review article by Faulkner (1974) for a somewhat different approach to these variables.

The fundamental theoretical problem of dwarf novae may be summarised as follows. Given that they all consist of short-period binaries in which mass transfer from a mainsequence component to a more compact (probably white dwarf) companion is taking place the question arises - are the outbursts due directly to mass transfer and the resulting accretion process, or are they due to the subsequent unstable nuclear burning of accreted material on the white dwarf surface? This central question - the exact cause of the outbursts - is still undecided, and it is this which will be our primary concern here.

Before examining the various physical processes that may be important we first summarise the fundamental features of dwarf novae in terms of their gross outburst properties, the underlying binary structure, and the detailed outburst behaviour.

\subsection{GROSS OUTBURST PROPERTIES}

The quasi-periodic outbursts exhibit a wide range of properties (see, for example PayneGaposhkin, 1957; Glasby, 1970). Glasby (1970) has presented a compilation of outburst light curves which demonstrate just how varied this behaviour can be. Clearly any account of the outbursts must be capable, at least in principle, of accommodating this variety. 
The eruptive behaviour of a typical system such as SS Cygni may be summarised as:

(1) A rapid initial rise to outburst maximum and slower decline. The rise time is frequently less than a day.

(2) A steady luminosity in the quiescent phase that occurs between outbursts.

(3) A total magnitude change of $\Delta m_{v} \sim 2.5-4 \mathrm{mag}$ during outburst.

(4) A typical mean outburst repetition period, $\tau \sim 30$ day. The outbursts are not strictly periodic, but repeat irregularly with extreme variations of a factor $\sim 2$ in $\tau$. The mean period varies from system to system, from $\sim 10$ days in a system like V485 Cen to $\sim 100$ days in $\mathrm{U}$ Gem.

(5) A remarkably low intrinsic luminosity, even at the extreme outburst stage. In the quiescent phase the absolute magnitudes deduced are generally consistent with the luminosity of a normal main-sequence star, possibly subluminous, but consistently a $\mathrm{G}, \mathrm{K}$ or $\mathrm{M}$ type dwarf. Thus values of $M_{\nu}$ (quiescent) $\sim+7.5\left(L \sim 3 \times 10^{32} \mathrm{erg} \mathrm{s}^{-1}\right)$ are typical, though values as low as $\sim+9.5\left(L \sim 4 \times 10^{31} \mathrm{erg} \mathrm{s}^{-1}\right)$ have been deduced for some systems (Strand, 1948; Kraft, 1962; Kraft and Luyten, 1965). At outburst the luminosity, at least in the optical region, seems to be limited to values of $M_{\nu}$ (outburst) $\gtrsim+3\left(L \lesssim 2 \times 10^{34} \mathrm{erg} \mathrm{s}^{-1}\right)$ (Warner, 1974a). The clear difference between the low luminosity character of dwarf nova eruptions and that of classical novae, which have outburst luminosities at maximum of the order of the Eddington limit for a solar mass object, i.e. $-6 \lesssim M_{v} \lesssim-8\left(L \sim 10^{38} \mathrm{erg} \mathrm{s}^{-1}\right)$ (Arp 1956; Payne-Gaposhkin, 1957; Finzi, 1973) should be noted.

In addition to these general features various idiosyncratic modes of behaviour are observed, both in individual systems and in certain dwarf nova subclasses. The occasional super maxima of systems like SU UMa and VW Hydri are in this category, as well as the standstills of the Z Cam subclass, and the unusual behaviour of AE Aqr - rapid fluctuations on a timescale of $10-30 \mathrm{~min}$ with an amplitude $\Delta m_{v} \sim 0.5 \mathrm{mag}$.

\subsection{BINARY STRUCTURE}

Following earlier work by Walker (1954), Joy $(1954,1956)$ and Crawford and Kraft (1956) it was finally shown in the classic work of Kraft $(1962,1963)$ that all observations of dwarf novae and classical novae were consistent with an interacting binary structure. According to this interpretation all dwarf novae consist of a red, apparently main-sequence, star which fills its Roche lobe, and transfers mass to an accreting blue component. The orbital periods of the dwarf nova binaries, $P \sim 12-2 \mathrm{~h}$ are all compatible with a main sequence, lobe filling, mass transferring star, and a white dwarf companion. in the classical novae and the recurrent novae, the red component is not necessarily a main sequence star, but is in some cases an evolved giant (e.g. T CrB with a binary period $\sim 230$ day).

Further details of the structure of dwarf nova binaries have been determined by Smak (1971a) and Warner and Nather (1971), and more recently Warner (1974b). A reasonably clear, though by no means simple, picture of their structure emerges from this important work. The blue component is surrounded by a flattened disc of accreted material. Matter is being fed into this disc by mass transfer from a red main sequence star. An optically bright hot spot is formed at the point where the mass transfer stream first interacts with the disc. The binary light curves of eclipsing systems generally exhibit a hump prior to eclipse which is attributed to the asymmetric radiation field (asymmetric with respect 
to the binary rotation axis) produced by the spot. The spot radiates only outwards from the disc, which is apparently optically thick. The eclipse itself, at least in U Gem, is interpreted as the eclipse of the spot only, and not of the accreting blue component or its surrounding disc (Warner and Nather, 1971; Smak, 1971a). Only in the case of Z Cha is the eclipse definitely that of the blue star and/or the disc (Warner, 1974b). For this reason this latter system has recently provided the most detailed information on the changes that occur during outburst.

\subsection{DETAILED OUTBURST BEHAVIOUR}

Ever since Kraft's determination of the binary structure of dwarf novae, the actual site of the outburst - the red star, the blue star, or the disc - has been difficult to ascertain straightforwardly. Krzeminski (1965) presented evidence, based on the eclipse behaviour of U Gem during outburst, that the red component was the dominant light source during eruptions. Walker and Chincarini (1968), however, interpreted their radial velocity measurements of SS Cyg as being contrary to such a model. Smak (1969) then showed that this radial velocity data could be interpreted as compatible with either the red or the blue component being the dominant luminosity source at outburst. Finally; in 1971, Smak and independently Warner and Nather, showed that the disc/hot spot model gave the most satisfactory account of the binary light curve properties. Krzeminski's conclusion was shown to be based on the erroneous (but natural) assumption that the eclipses in the UGem light curve were eclipses of the blue component. If instead the eclipse was that of a stream/disc hot spot as Smak (1971a) and Warner and Nather (1971) suggested then the blue component or the disc could be the dominant light source at outburst. The shallow eclipse depth at outburst then only implied that the hot spot had not brightened significantly, not that the disc or blue component were unchanged. Smak went on to suggest that it was the disc itself which was brightening during outburst in U Gem, and summarised possible outburst mechanisms as either due to -

(1) an instability in the outer layers of the primary blue component;

(2) periodic unstable mass outflow by the red star and subsequent brightening of the disc;

(3) instabilities within the disc itself.

These general conclusions were dramatically confirmed by Warner's (1974b) observations of Z Cha. This system, found by Mumford (1969) to be an eclipsing binary with a 107-min period, was discovered by Warner to suffer large changes in primary eclipse shape during outburst. In the quiescent state rapid ingress and egress, producing a $U$ shaped primary eclipse, occurs. At outburst this develops into a broader and deeper (in absolute energy units) $V$ shaped eclipse. Warner showed that these observations could be fitted straightforwardly with the following sequence of events. In the quiescent phase the disc itself does not appear to radiate strongly except possibly at the inner disc/white dwarf boundary. Thus the quiescent state may be described as consisting of the blue component (or disc boundary), the hot spot and the red component. Durnng outburst the whole disc starts to radiate out to a radius of $\sim 10^{10} \mathrm{~cm}$. The luminosity of the disc increased by $\sim 2.5$ mag above the background luminosity contributed by the other components. In this state the position and brightness of the hot spot (or rather the binary light curve shoulder) are variable. Similar hot spot behaviour had already been noted by Smak (1971b) and disc changes had been commented on by Paczynski (1965a), Robinson 
(1973), Smak (1971a) and Warner and Nather (1971). However, Warner's (1974b) observations provide not only conclusive evidence, but direct, quantitative measures of the changes that are occurring.

In addition to this important work on the precise outburst behaviour, a whole new field of research was opened up by Warner and his group (Warner and Robinson, 1972; Warner, 1973; Robinson, 1973; Warner, 1974b; Warner and Harwood, 1973; Warner and Brickhill, 1974) with the discovery of transient, low-amplitude periodicities at outburst. These periodicities are sinusoidal, to the limits set by the background noise, with periods in the range $17-35 \mathrm{~s}$. Originally they were interpreted as due to pulsations of the underlying white dwarf and discussed by Warner and Brickhill (1974) and Brickhill (1975), but there may be energy problems with such an interpretation, though these may not be insuperable if surface waves are proposed rather than global pulsations. Bath (1973) suggested that the pulses could be produced by the periodic eclipse of inhomogeneities, or fluctuations, in the innermost disc region as they orbit the accreting white dwarf. Another possibility might be a shot-noise model, originally proposed by Terrell (1972) to account for the somewhat similar periodicities observed in certain X-ray sources. The correct explanation of these periodicities is not yet clear, but they could contain a wealth of undeciphered information about dwarf novae outbursts.

Turning now to the problem of physical interpretation, the question remains which of the three possibilities suggested by Smak is valid in the light of these observations, particularly those of Z Cha. Warner interpreted his results in terms of a nuclear burning model suggesting that the disc brightening occurred as a result of a strong shock propagating out through a massive disc $\left(\sim 10^{-4} M_{\odot}\right)$. Starrfield et al. (1974) independently suggested that such behaviour could be occurring in dwarf novae. However, Bath et al. (1974) have shown that such shock propagation is difficult to achieve in a centrifugally supported disc, due to the decreased importance of pressure support. Furthermore it should be stressed that none of the nuclear burning calculations published so far has yet reached the stage of obtaining behaviour which with reasonable (or even unreasonable) initial conditions in any way resembles the outburst amplitudes, timescales, repetition periods, energies, or disc confinement observed in dwarf novae - and all implicitly ignore the luminosity contributed by accretion of infalling matter.

The second possibility of Smak in which this accretion energy plays the dominant role was also considered by Bath (1973) and was examined in detail by Bath et al. (1974) for the case of $\mathrm{ZCha}$ about which so much data is available. In this latter case it was shown that the observed behaviour of $\mathrm{Z}$ Cha was just that that would be expected for a modulated mass transfer, accretion model.

This model will now be reviewed and two points should be stressed. Firstly the results of Section 3 and 4 result from a general theoretical treatment of the stability and accretion disc properties of interacting binaries. If these results are in error then something is fundamentally wrong with techniques that are used, none of which are especially original or non-standard. Secondly, it should be noted that many of the features that are discussed must also be important in other interacting, semi-detached binaries, though because of their different binary parameters (masses/radii/luminosity etc. of both components) they will manifest themselves in different ways in other systems.

The problem of binary star stability, and the accretion processes that result from mass transfer, pose difficult, but important, scientific problems, most of which have only been 
fully appreciated in the past two decades. The whole subject is in an exciting state of flux and development.

\section{Energy Sources}

In a semi-detached binary in which mass transfer is occurring, the mass transfer process itself results in energy sources being present. To illustrate this consider for simplicity a steady flow, $\dot{m}$, between the red and blue components. At each point within the flow acceleration in the surface of the red component, infall onto the blue component, and final assimilation by the blue component - various energy sources can contribute to the resulting luminosity of the system.

Starting on the blue component and working backwards along the flow, the most energetic source is evidently the nuclear burning energy that is available if the blue component is a degenerate white dwarf. It is this high efficiency of nuclear burning that has led to the widespread belief that nuclear burning instabilities would account for dwarf nova eruptions in a similar way to the classical nova models discussed by Starrfield et al. (1976) and references therein. Therefore the energy per gram available after sufficient material has been accreted to produce ignition conditions is $\sim 5 \times 10^{18} \mathrm{erg} \mathrm{gm}^{-1}$. In a steady state, with burning proceeding on average at the same rate as accretion, the resulting nuclear luminosity is $L_{n} \sim 5 \times 10^{18} \dot{m} \mathrm{erg} \mathrm{s}^{-1}$. Clearly rather low values of $\dot{m} \sim 10^{15}$ $10^{16} \mathrm{gm} \mathrm{s}^{-1}$ could account for outburst luminosities of $\sim 10^{34} \mathrm{erg} \mathrm{s}^{-1}$, and an average value of $\dot{m}$ less than this would be necessary to account for the quiescent phases unless bolometric corrections are very important. We must conclude that nuclear burning provides an abundant supply of energy in dwarf novae for the normally-suggested mass transfer rates of $10^{16}-10^{18} \mathrm{gm} \mathrm{s}^{-1}$. We stress however that there are more problems than just providing an energy source. The source must be capable of generating optical outburst luminosities of $\sim 10^{34} \mathrm{erg} \mathrm{s}^{-1}$ on timescales of $\sim 1$ day, and relax and repeat on timescales of 10-100 days.

Two rather different methods of studying nuclear burning instabilities have been considered. The first starts with unstable initial conditions and follows the subsequent hydrodynamic behaviour of the outbursts. Starrfield et al. (1972) have shown that, given a sufficient choice of initial conditions, such models can give sudden outbursts, similar to classical novae, with runaway to approximately Eddington limit luminosities.

The second approach considers the slow growth of a spherically-symmetric accreted envelope and follows, non-hydrodynamically, the development of the unstable burning that eventually occurs. In Figure 1 the resulting luminosity variations of four typical models of this type are given, according to the results of Giannone and Weigert (1967), Redkoborodyi (1972), and Taam and Faulkner (1975). Note that the growth time of the instability is typically $10^{4}-10^{5} \mathrm{yr}$. Furthermore in no case have steady conditions been achieved. The surface luminosity, $L_{n}$, is always very much less than $5 \times 10^{18} \dot{m}$. The final evolution of such systems remains an unexplored and interesting problem. There are reasons to believe that in almost all cases such runaways will eventually lead to phases in which the luminosity is of the order of the Eddington limit luminosity.

Present understanding of the nuclear burning problem has not solved the problem of dwarf novae. If it is to explain their eruptive behaviour it must also, of course, explain the disc brightening that is observed in $\mathrm{ZCha}$, and the cause of the large differences 


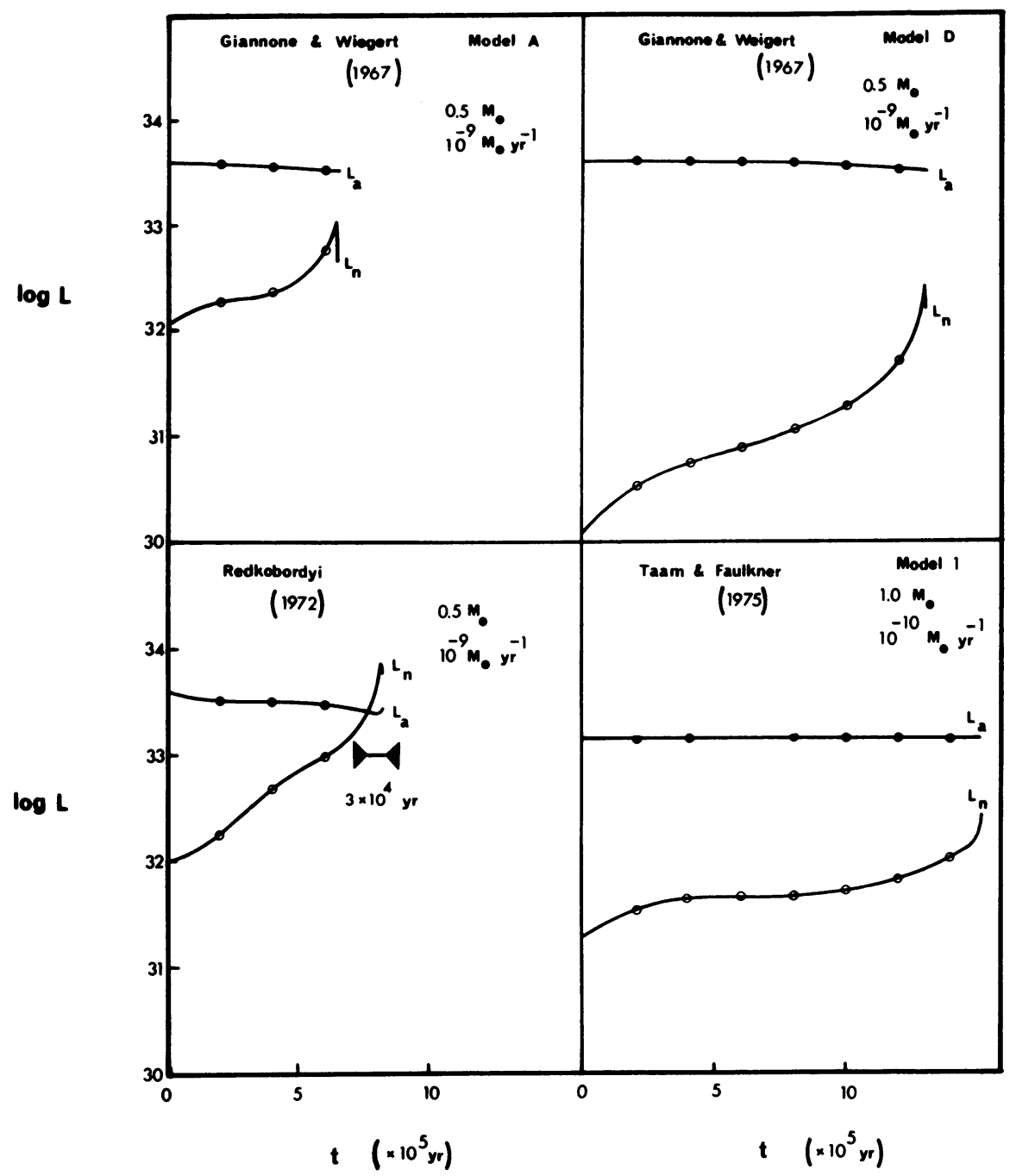

Fig. 1. Variation of surface luminosity due to nuclear burning, $L_{\mathrm{n}}$, and accretion luminosity, $L_{\mathrm{a}}$, in four typical accreting white dwarf models. In only one case is $L_{n}$ greater than $L_{a}$ for the phases treated. The values of $L_{\mathrm{a}}$ at these mass transfer rates are typically the same as the mean dwarf nova luminosities observed.

between dwarf novae and classical novae. In pointing out these present failures of nuclear burning we do not intend to imply that nuclear burning can be discounted as wholly irrelevant. It is important at some stage, if the blue component is indeed a white dwarf perhaps either in producing an evolutionary transformation of the system, or in occasionally producing periodic classical-nova eruptions.

The second energy source is the accretion energy dissipated in the accretion disc. The energy available per gram from this source is just the potential energy of infall, $G M_{1} / R_{1}$. This provides a luminosity, $L_{a} \sim 10^{17} \mathrm{erggm}^{-1}$ for a white dwarf companion of $1 M_{\odot}$ 
Accretion is 50 times less efficient than nuclear burning, but for accretion rates of $10^{16}$ $10^{18} \mathrm{gm} \mathrm{s}^{-1}$ it still provides an abundant energy supply. Furthermore this accretion energy will be liberated within the accretion disc, at exactly the site observed in $\mathrm{Z}$ Cha. To illustrate the relative importance of this infall energy, the accretion luminosity of all four nuclear burning models is given in Figure 1. Not only has the surface nuclear luminosity, $L_{n}$, never reached steady-state values of $5 \times 10^{18} \dot{m}$, but in only one case is it ever greater than the accretion luminosity, $L_{a}$, and then only marginally so.

We conclude that accretion can in principle provide an abundant enough energy supply to power dwarf nova outbursts. One only requires the accretion flow to be modulated in quasi-periodic bursts, either within the disc itself, or during the original injection by the red component. Osaki (1974) has considered the former possibility. Here we will examine modulation of the mass transfer rate at its origin, that is within the red component envelope.

This brings us to the third energy source. The red component is consistently a cool $\mathrm{G}, \mathrm{K}$ or $\mathrm{M}$ type dwarf. Mass transfer from such stars must result in the recombination of ionized material below the photosphere, as it passes through the ionization zones. As a result $\sim 10^{13} \mathrm{erg} \mathrm{gm}^{-1}$ become available in the flow at this point. This third energy source acts in two ways. Firstly it may destabilize the red component envelope, preventing hydrostatic equilibrium conditions from being achieved, and driving dynamical instabilities. Secondly it can contribute to the overall luminosity of the system by increasing the effective luminosity of the red component. It is now clear that the second effect is negligible in comparison with the accretion luminosity. However the destabilizing influence of the ionization zones may be crucial in modulating the mass transfer rate to produce bursts, or hiccoughs, of mass transfer, with an outburst period of $\sim 30$ day in low mass, main sequence, red components.

\section{Stability of the Red Component}

Mass loss and subsequent transfer by the contact component of a semi-detached system has been studied from several different points of view (see, for example, Paczynski, 1971). Here we consider only the dynamical effects that may occur in the red component, and ignore long term evolutionary problems.

Recent work at Oxford on the dynamical stability of mass-transfering binary members has been based on two rather different developments of the earlier approaches of Bath $(1969,1972)$. The first of these examines the linear stability of model envelopes that have been constructed within the correct Roche potential, rather than in spherical symmetry (see Papaloizou and Bàth, 1975). These models are constructed using a normal mixing length treatment of convection and with their photospheres sufficiently below the critical Roche lobe to allow an atmosphere to be accommodated. The stability of models both along, and off, the line of centres of the binary has been analyzed using standard variational principle procedures. The main conclusions of this work may be summarized as follows:

(1) Models in the low-mass main-sequence region of the HR diagram (providing they are not of too low mass) and red giant models are normally unstable.

(2) The instability is confined to a small cone about the line of centre, with a cone 
angle which is subtended by approximately a local scale height, $H_{\mathrm{L}}$, at the Lagrangian point, where

$$
H_{\mathrm{L}} \sim \frac{1}{2}\left(\mathscr{R} T_{\mathrm{s}} R_{\mathrm{s}}^{3} / G M\right)^{\frac{1}{2}}
$$

$T_{\mathrm{s}}$ and $R_{\mathrm{s}}$ are the surface temperature and radius (see Figure 2).

(3) From a linear point of view two effects can contribute to destabilization. One is the degree of superadiabaticity of the envelope. This term is generally dominated by the

\section{(a)}

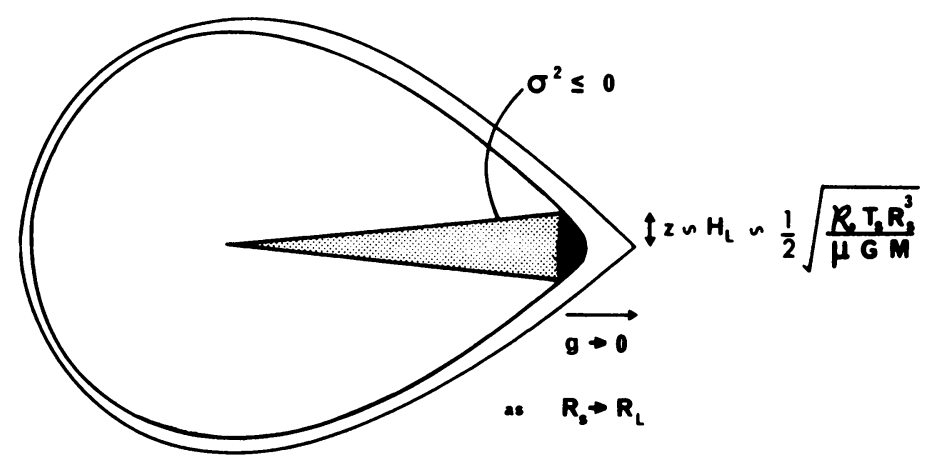

(b)

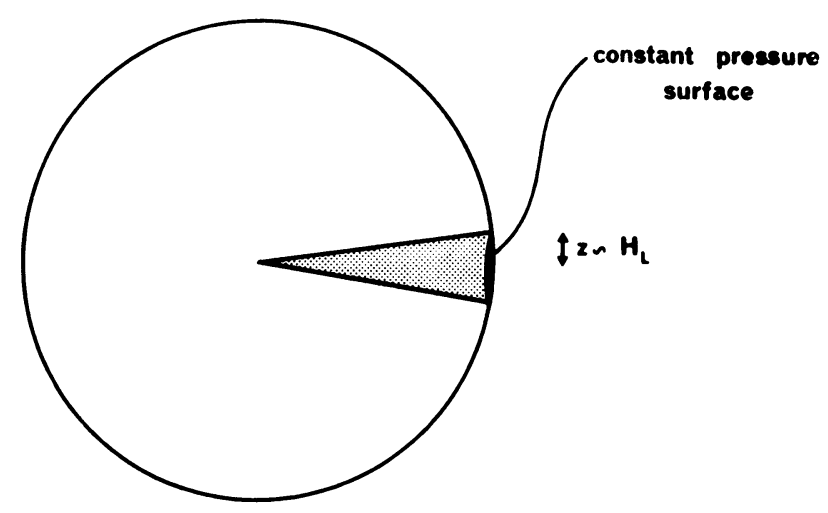

Fig. 2. (a) The stability behaviour of Roche models. With the photosphere (along the line of centres) approximately one scale height, $H_{\mathrm{L}}$, below the critical point, a cone subtended by $\sim H_{\mathrm{L}}$ is unstable $\left(\sigma^{2} \leqslant 0\right)$. Sequences of models with decreasing photospheric radii suffer a decrease in this cone angle, eventually shrinking to the line of centres and finally, when sufficiently detached, the whole envelope becomes stable.

(b) Schematic representation of the non-linear scheme. Spherical models are considered, but outflow is restricted to a cone angle of the same size as that suggested by the Roche analysis. 
strong superadiabatic gradients in the ionization zones, and from an energy point of view this is identical to the effect of recombination discussed in Section 2. The second destabilizing influence is the variation in the gravitational potential that arises through mass redistribution in the star during mass loss. This second effect accounts for the polytropic instabilities discussed by Paczynski (1965b).

We conclude that any attempt to construct a semidetached system with such unstable components as contact members must result in some form of dynamical outflow in the vicinity of the Lagrangian point.

The second treatment attempts to model the nonlinear hydrodynamic development of these instabilities (Bath, 1975). The procedure adopted in this work treats spherical models using standard hydrodynamic difference techniques. The numerical code allows the behaviour of the envelope to be followed in both a mass-conserving Lagrangian coordinate scheme, and in a mass-losing, but radius conserving, Eulerian coordinate scheme. If the stellar photosphere is less than the critical Roche radius the former state is assumed, and the envelope may freely expand or contract. If the envelope fills the Roche lobe, mass loss is permitted, and a boundary condition is imposed on the photosphere which introduces the effect of zero gravity at the Lagrangian point. Convection is necessarily ignored. We summarize below the behaviour of such models.

(1) In the contact state the envelopes of stars cool enough to possess ionization zones are unstable. Mass outflow builds up on a dynamical timescale, reaching outflow velocities of the order of the sound speed. After reaching a maximum value the mass loss rate, $\dot{m}$, slowly decreases and eventually halts. This behaviour is shown in Figure 3, where $\dot{m}$ has been plotted as a function of time for three zones in the envelope at differing depths (zone 34 is the photosphere). The instability is driven by recombination energy liberated in the ionization zones. Outflow eventually ceases when this recombination energy is insufficient to lift further material through the gravitational potential to the surface. Thus the outflow stops when matter comes to the surface at radius $R_{\mathrm{L}}$ which was originally at a depth $\Delta R$ given approximately by,

$$
\Delta R \sim \frac{R_{\mathrm{L}}{ }^{2} I}{G M}
$$

where $I$ is the recombination energy per gram $\left(\sim 10^{13} \mathrm{erg} \mathrm{gm}^{-1}\right)$

(2) At the stage when outflow ceases the envelope is far from thermal equilibrium. Introducing the switching procedure to a Lagrangian formulation at this point when inwardly directed velocities first appear at the surface, the envelope contracts away from the lobe and then slowly readjusts to thermal equilibrium. The luminosity deficit that has developed is slowly restored by energy transport from the interior, and the photosphere gradually expands back towards the critical Roche surface. Eventually, on a timescale given approximately by the thermal timescale of

$$
\tau_{\text {th }} \sim \frac{\mathscr{R} \bar{T} \Delta M}{\Delta L}
$$

where $\Delta L$ is the average luminosity deficit in the envelope of mass $\Delta M$, and mean temperature $\bar{T}$, the photosphere regains the contact state and a second outflow instability occurs. This sequence of repeated outbursts by the red star is shown for a typical model 


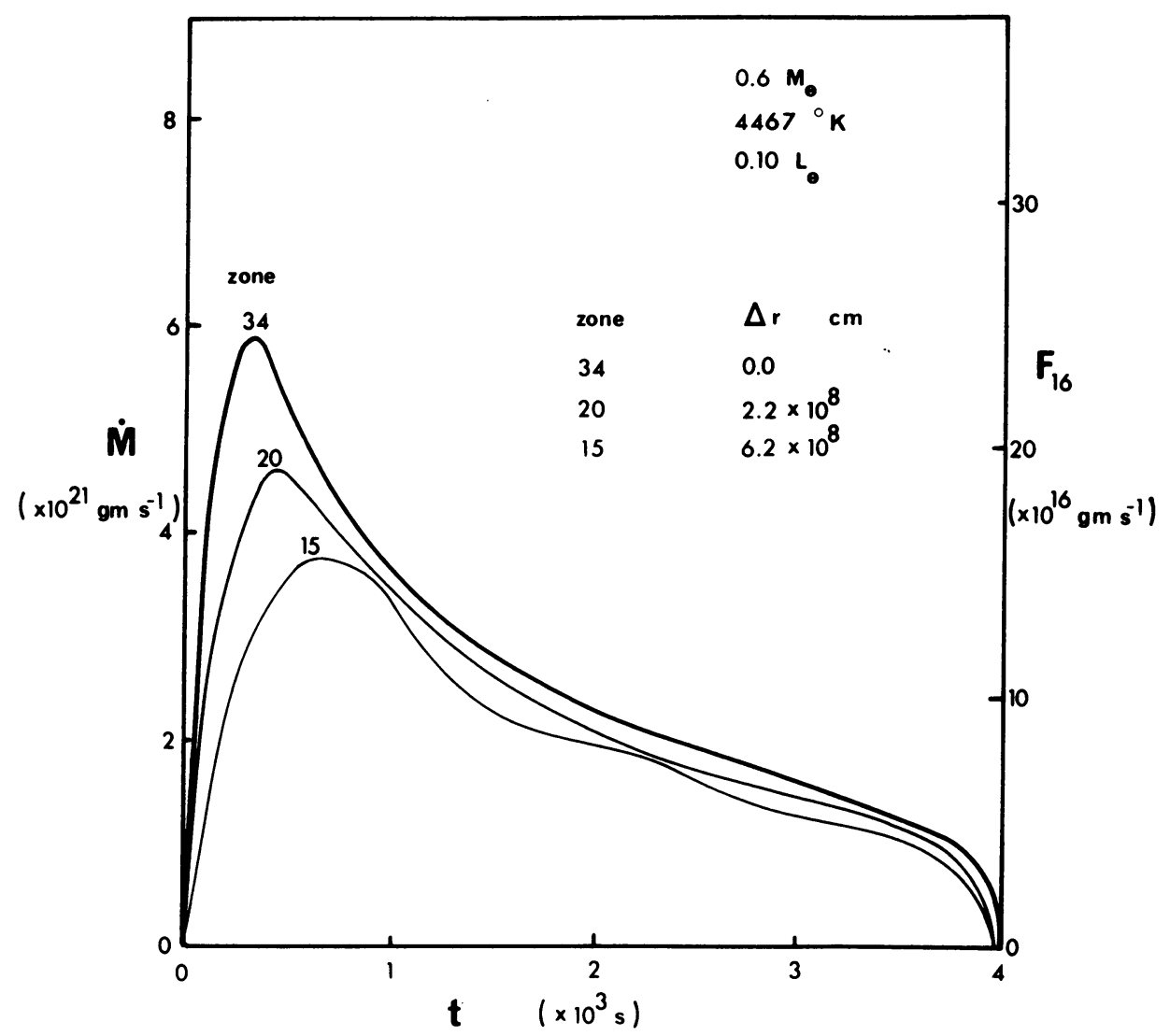

Fig. 3. Variation of $\dot{m}$ through three zones in the mass outflow phase as a function of time. Zone 34 is the photosphere. $\dot{M}$ is the spherical outflow rate, $F_{16}$ the reduced outflow rate in a cone which has scale height dimensions at the surface.

in Figure 4. The timescale between such outbursts is typically 10-200 days in the lower main-sequence models.

(3) These spherical models must be interpreted carefully since the Roche models discussed previously suggest that dynamical outflow only occurs in a small cone about the line of centres of the two stars. This effect was not considered in the earlier spherical work of Bath $(1969,1972)$. The simple though by no means rigorous procedure that we have adopted to account for this reduction is to restrict the spherical outflow to a cone of the same dimensions as that which is suggested by the linear analysis of Roche models (Figure 2). When this is done the mass transfer rates are severely reduced. We shall refer to these reduced mass-transfer rates with the symbol, $F$, rather than $\dot{m}$, and work in units of $F_{16}=10^{16} \mathrm{gm} \mathrm{s}^{-1}$. Then the outflow rates during outburst are consistently $F_{16} \sim 10-10^{3}$, except for the red giant models when $F_{16}$ may be as high as $10^{4}$.

We conclude from this work (the reader is referred to the original papers for details) that the red components of dwarf novae are unstable. The instabilities result in periodic outbursts during which large, enhanced values of the mass transfer rate occur. Of course 


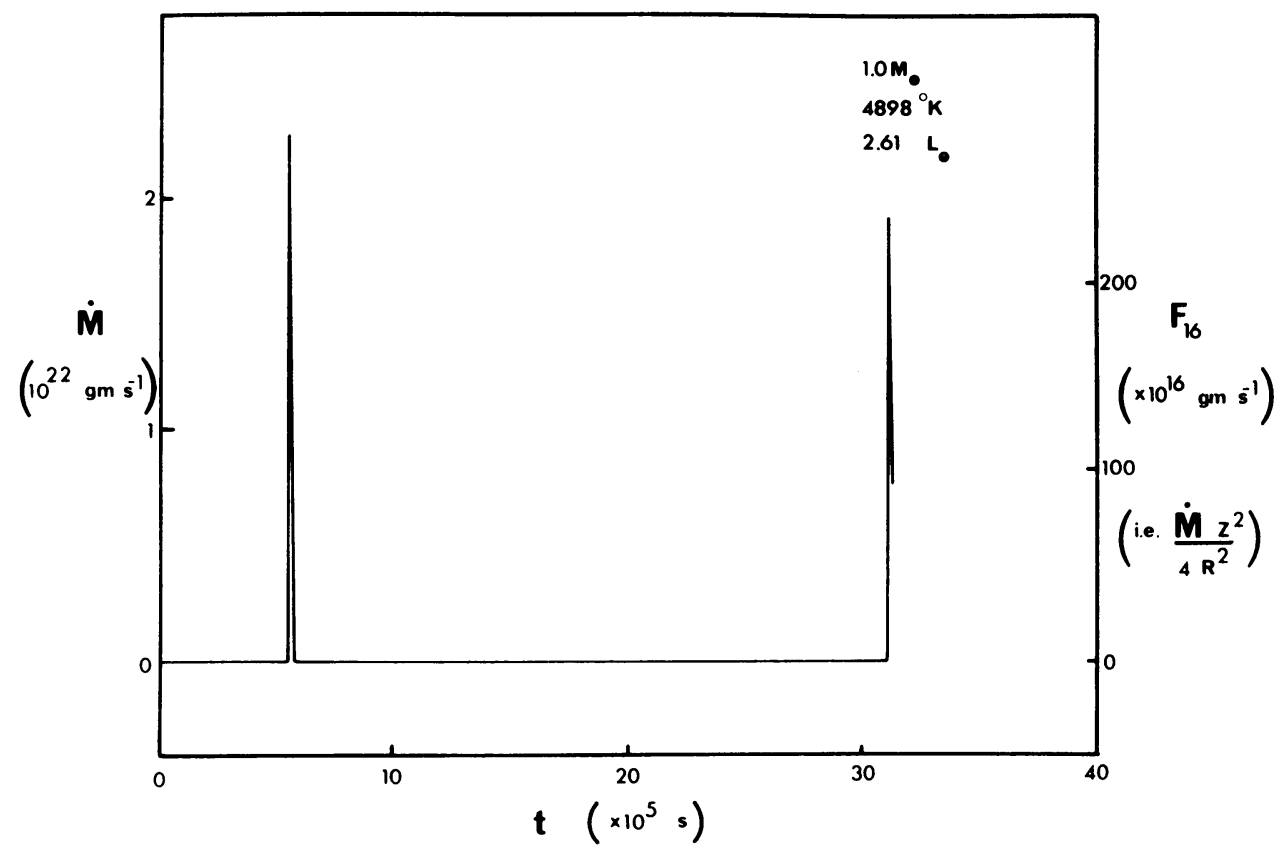

Fig. 4. Two outbursts of a typical spherical model. Between such outbursts the photosphere contracts below the Roche lobe with the envelope readjusting back to a state of thermal equilibrium.

in the quiescent stage some mass transfer will continue, simply as a result of a solar-wind type outflow. The mass transfer rate rises to $F_{16} \sim 10-10^{3}$ at outburst, and outbursts repeat with a period which is typically the same as the outburst period observed in dwarf novae.

\section{Disc Properties}

After such dynamically unstable bursts of enhanced outflow by the red component, the question immediately arises - how does the disc respond to this increased flux of matter in the mass transfer stream? This problem has been discussed previously from the point of view adopted here by Bath et al. (1974) and Evans (1974) for the particular case of the system $\mathrm{Z}$ Cha. Here we demonstrate that the gross properties of dwarf novae as a class can be accounted for in terms of such a model.

In the model of $\mathrm{Z}$ Cha by Bath et al. (1974) the disc properties were discussed on the basis of steady state accretion. The models of accretion discs developed in the X-ray binary context by Pringle and Rees (1972) and Shakura and Sunyaev (1973) were applied to the case of white dwarf accretion, and the optical appearance of such discs determined. Here the $U, B, V$ and soft X-ray appearance of such discs, accreting onto white dwarf companions, are discussed.

The temperature distribution, $T(R)$, as a function of radius, $R$, in such a steady state disc is given by (Shakura and Sunyaev, 1973), 


$$
T(R)=\left(\frac{3 F G M_{1}}{8 \pi \sigma R_{1}}\right)^{\frac{1}{4}} X^{-\frac{3}{4}}\left(1-X^{-\frac{1}{2}}\right)^{\frac{1}{4}}
$$

where $X=R / R_{1}, R_{1}$ is the inner radius and $F$ is the accretion rate. This relation ignores the boundary layer problem that exists at the white dwarf surface. In one attempt to account for this Novikov and Thorne (1973) introduce a parameter $\beta$ in the last term (i.e. $\left.\left(1-\beta X^{-\frac{1}{2}}\right)^{\frac{1}{4}}\right)$. If $1>\beta>0$ then the disc does work in accelerating the accreting component and if $\beta<0$ then the dwarf does work on the disc.

The intensity of the disc at any wavelength can be derived on the assumption that each element radiates as a black body. Then

$$
I_{\lambda}=\int_{R_{1}}^{R_{\mathrm{d}}} \pi B_{\gamma}(T(R)) 2 \pi R \mathrm{~d} R
$$

where $R_{\mathrm{d}}$ is the outer disc radius and $B_{\lambda}(T)$ the radiation intensity per unit wavelength range for a black body at temperature $T$. For the conditions that we consider the optical depth through the disc is in general greater than unity (Pringle and Rees, 1972; Shakura and Sunyaev, 1973; Evans, 1974) and the assumption of black body radiation by each annulus is approximately valid. However the effects of line emission will become important at low accretion rates when temperatures drop below $10000 \mathrm{~K}$ according to a black body assumption. In our case the inner radius of the disc, $R_{1}$, will be $\sim 10^{9} \mathrm{~cm}$ and the outer radius must be less than the Roche lobe size $\left(\lesssim 10^{11} \mathrm{~cm}\right)$. In the following we consider values of $1,1.4$ and $5 \times 10^{10} \mathrm{~cm}$, and mass fluxes of $10^{14}-10^{21} \mathrm{gm} \mathrm{s}^{-1}$ onto a solar mass white dwarf.

The spectrum of the disc has been obtained by integrating numerically the contribution of each annulus. These have then been fitted with the response curves of $U B V$ filters, and the $U B V$ appearance at different accretion rates determined. In Figure 5 the resulting $V$ magnitudes are given for different values of $\beta$. Since $\beta$ only affects the temperature distribution significantly in the inner region, which is generally in the RayleighJeans limit at optical wavelengths, the $U B V$ appearance is not very sensitive to $\beta$. No inclination effects are included. The bolometric magnitudes are shown for comparison. Three conclusions may be drawn immediately.

(1) The quiescent state of dwarf novae can be accounted for by accretion at rates $\sim 10^{16} \mathrm{gm} \mathrm{s}^{-1}$ if the accretion disc dominates the optical luminosity at this phase. The visual magnitude of the disc is then $M_{v} \sim+8$, the typical quiescent magnitude of dwarf novae. If the red component is the dominant source then $F$ could be less. than this (though the hot spot contribution could still be significant in this state, as is generally observed). In this quiescent state almost all the disc energy is radiated at optical wavelengths.

(2) Outbursts corresponding to a 3-mag increase in $V$ may be produced by enhanced mass transfer at $F \sim 10^{18} \mathrm{gm} \mathrm{s}^{-1}$. At this rate $M_{v} \sim+5.5$ whilst $M_{\text {bol }} \sim+2.5$. Such outbursts would be bluer than the quiescent state (as observed - Paczynski, 1965b) and a considerable fraction of the luminosity would be radiated in the ultra-violet region. We emphasise that the mass transfer rates required at outburst are relatively low, corresponding to $10^{-8} M_{\odot} \mathrm{yr}^{-1}$. We further note that this value is approximately that that the hydro- 


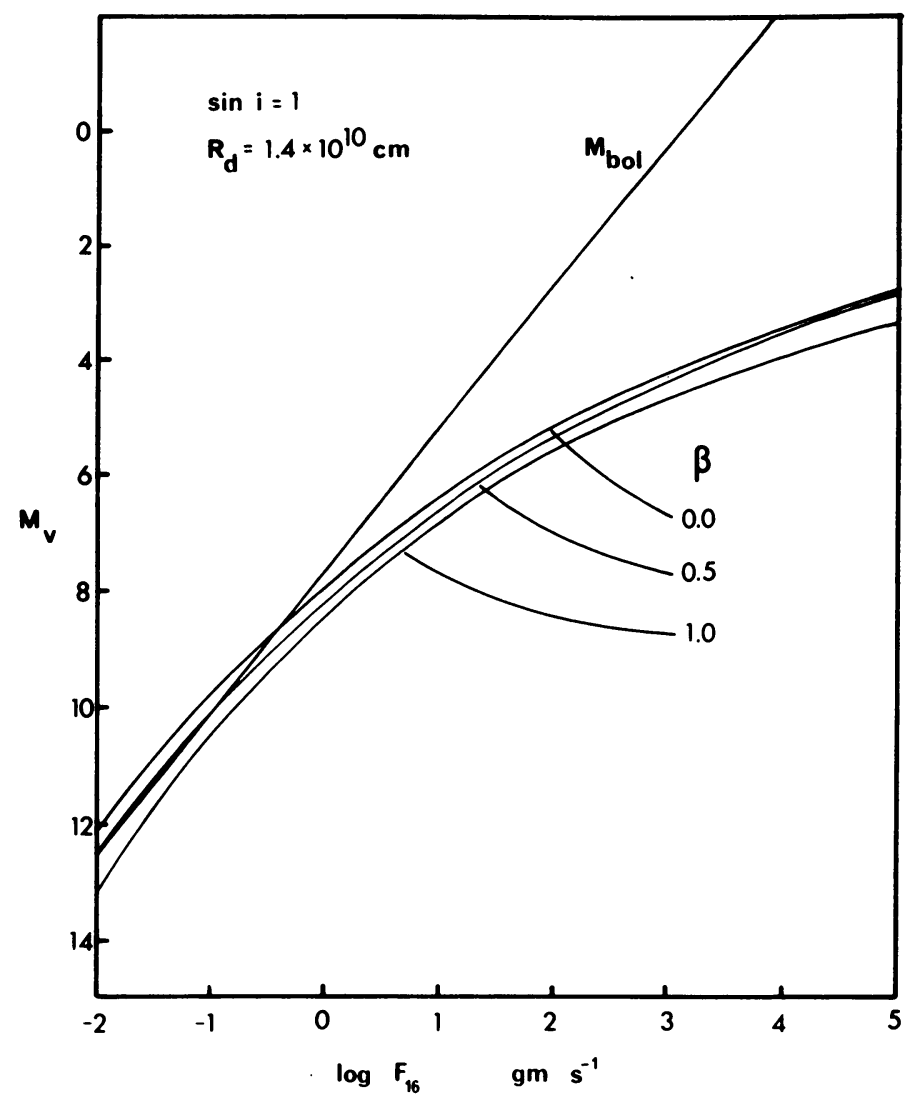

Fig. 5. $\quad V$ magnitude of a steady state disc at differing accretion rates, together with the corresponding bolometric magnitudes.

dynamic models of red component instabilities suggest if the outflow is confined to a region of scale height dimensions at the inner Lagrangian point (section 3).

(3) For accretion rates that generate a total luminosity that is less than the critical Eddington limit value (which occurs at $F_{16} \sim 10^{5}$ ), at which stage the accretion disc is probably disrupted by radiation pressure (Shakura and Sunyaev, 1973) the visual magnitude is never greater than +3 mag. Variations in $R_{\mathrm{d}}$ can affect this value marginally (see Figure 6), but $M_{v}$ must be of (or less than) this order at outburst. Note that the estimated observed maximum magnitude attained by dwarf novae at outburst has exactly this value (Warner, 1974a). The consequences of accretion at rates greater than the Eddington limit form a separate problem. Bath et al. (1974) have suggested that it may be related to classical novae outbursts.

Figure 6 presents visual magnitudes for the two components - disc plus red component. We ignore the contribution of the impact hot spot at the disc edge, and any intrinsic luminosity of the white dwarf. Four main sequence components of spectral type G5 to M0 (corresponding to masses $0.93,0.78,0.69$ and 0.47 respectively - Allen, 1973) and two discs with outer radii $R_{\mathrm{d}}=1 \times 10^{10} \mathrm{~cm}$ and $5 \times 10^{10} \mathrm{~cm}$ are shown. 


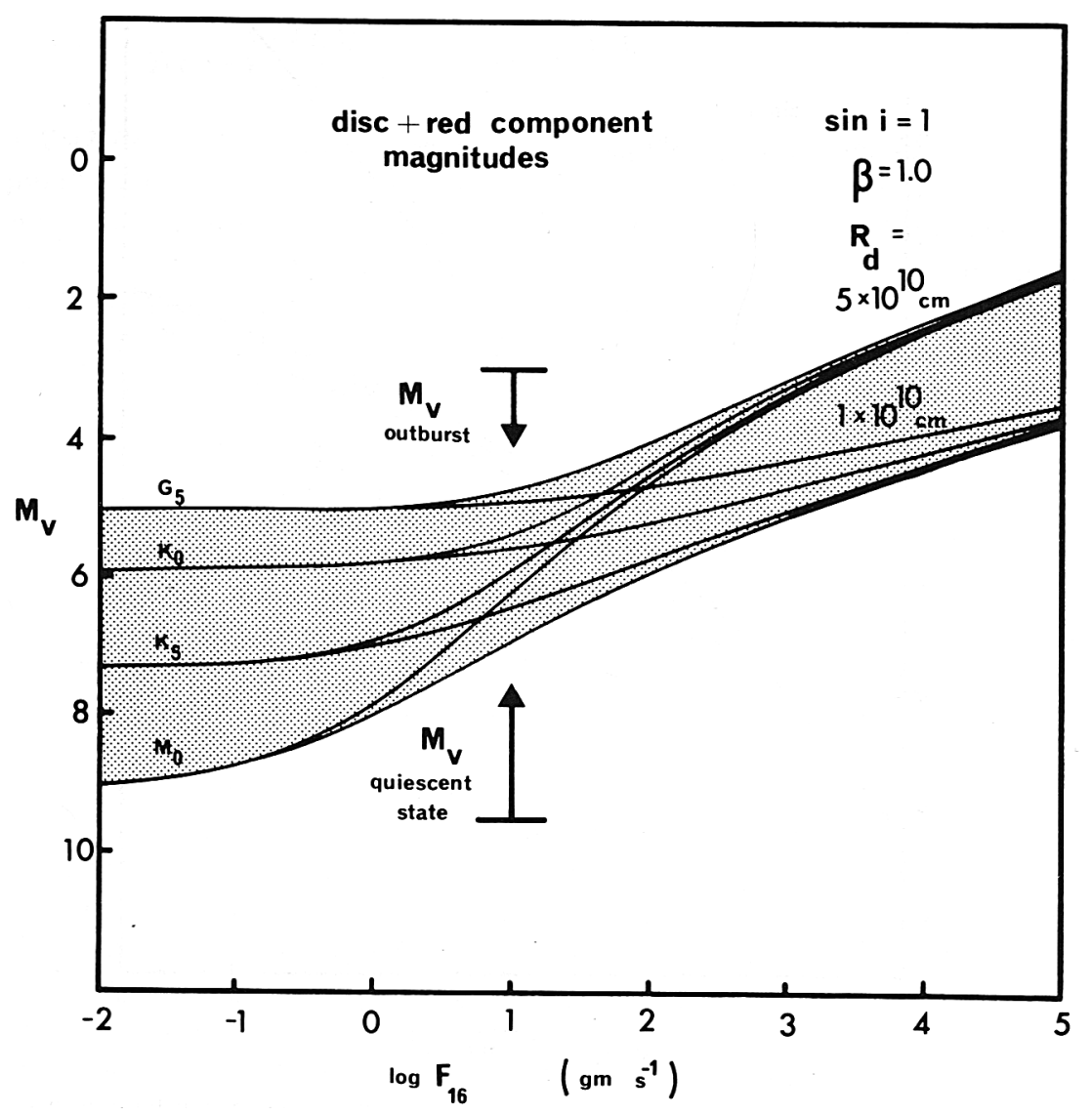

Fig. 6. Total $V$ magnitudes of the system - red component plus disc - for four red main sequence stars and two discs of different outer radii at different accretion rates. An indication of the minimum visual magnitude observed in dwarf novae in the quiescent state $(+9.5)$ and the maximum visual magnitude observed in dwarf nova outbursts $(+3)$ is shown.

Consideration of the dimensions of a binary in which the red star is a contact, lobe filling component suggest that disc radii $\sim 1 \times 10^{10} \mathrm{~cm}$ may be generally correct for systems containing $\mathrm{M} 0$ red components, and $\sim 5 \times 10^{10} \mathrm{~cm}$ for $\mathrm{G} 5$ components.

It is clear from this figure that outbursts of several magnitudes in the visual would be produced by enhanced mass transfer. A G5 component transferring mass at a rate $\sim 10^{19}$ $\mathrm{gm} \mathrm{s}^{-1}$ would increase the visual luminosity of a disc of radius $R_{\mathrm{d}} \sim 5 \times 10^{10} \mathrm{~cm}$ by two magnitudes above that of the red component. A system containing an M0 component transferring mass at a rate $\sim 10^{18} \mathrm{gm} \mathrm{s}^{-1}$ with $R_{\mathrm{d}} \sim 1 \times 10^{10} \mathrm{~cm}$ would be three magnitudes brighter than the red component alone. The large disc contribution which is responsible for the increase in $M_{v}$ in these systems at a mass transfer rate of $F \gtrsim 10^{16}$ $\mathrm{gm} \mathrm{s}^{-1}$ demonstrates just how important mass transfer, and the associated liberation of accretion energy, can be in these systems.

The form of the optical eclipse curves of such accretion discs has been considered by Bath et al. (1974). In Figure 7 and 8 the $U B V$ eclipse curves are given for mass accretion 


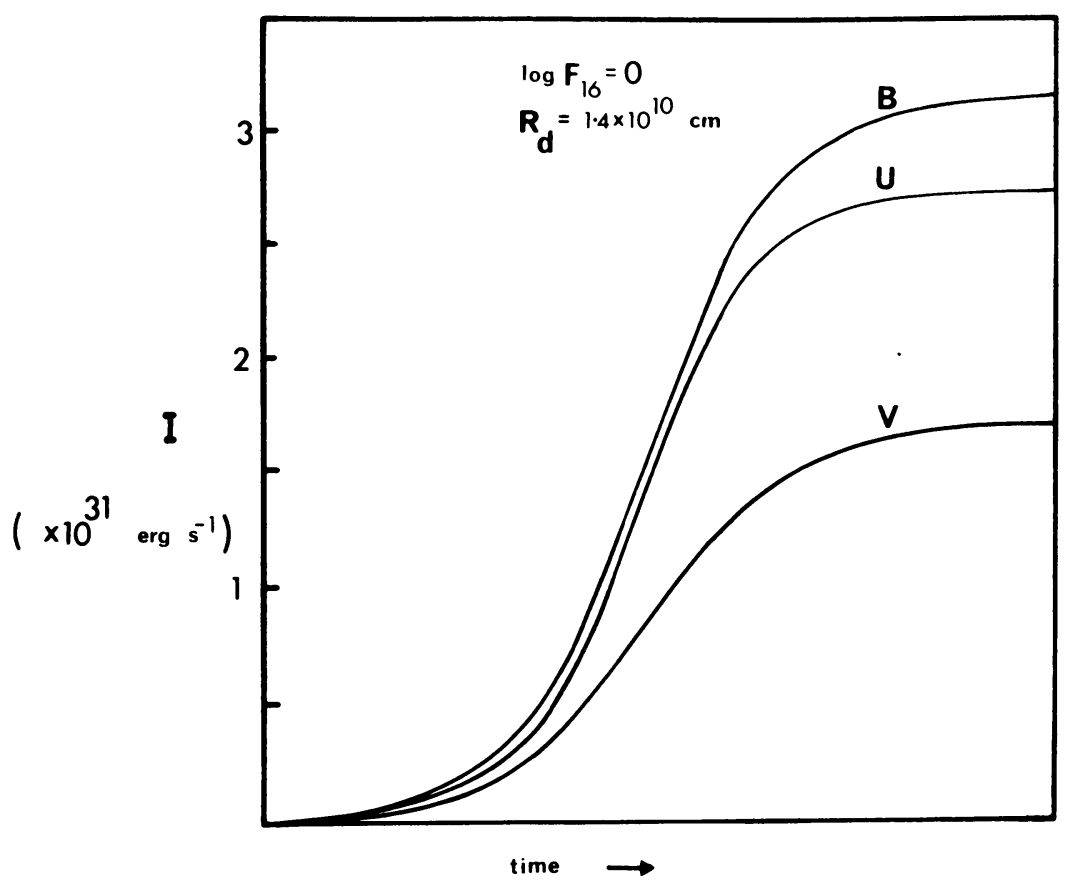

Fig. 7. Eclipse of a steady disc at $F_{16}=0$ (low accretion rates). A sharp, $U$-shaped eclipse occurs, since the optical luminosity is dominated by the central disc regions.

rates of $10^{16}$ and $10^{18} \mathrm{gm} \mathrm{s}^{-1}$ for straight edge eclipses. A change from $U$ to $V$ shaped form during outburst is apparent, similar to the changes observed by Warner (1974b) in $\mathrm{Z}$ Cha. These changes are simply due to the changes in temperature associated with the changing accretion rates. At low accretion rates the temperatures are such that most of the radiation is in the optical region and the intensities are approximately proportional to $T^{4}$. At high accretion rates the optical region moves into the Rayleigh-Jeans limit for most of the disc and the intensities are proportional to $T$. The temperature distribution of the disc is in this way reflected in the eclipse curves. These are dominated by the centre of the disc at low mass transfer rates and become much more extended at high mass transfer rates. This behaviour illustrates the important point that in the quiescent state the luminosity which is generally ascribed to the blue component may be that of the disc alone.

So far we have ignored the important effects of disc inclination to the observer's line of sight. The reduction in luminosity due to disc inclination, due both to geometrical and limb darkening effects, will be important for all cases in which $\sin i$ is significantly less than unity (Bath et al., 1974). However any period/amplitude relationship for the outbursts (such as the Kukarkin and Parenego relationship) must suffer large scatter due to inclination effects alone.

The mass transfer rates at outburst could become sufficiently large, in some circumstances, to generate a detectable soft X-ray flux. However the soft X-ray flux will be generated in the Wien region of the spectrum, and will therefore be extremely sensitive to 


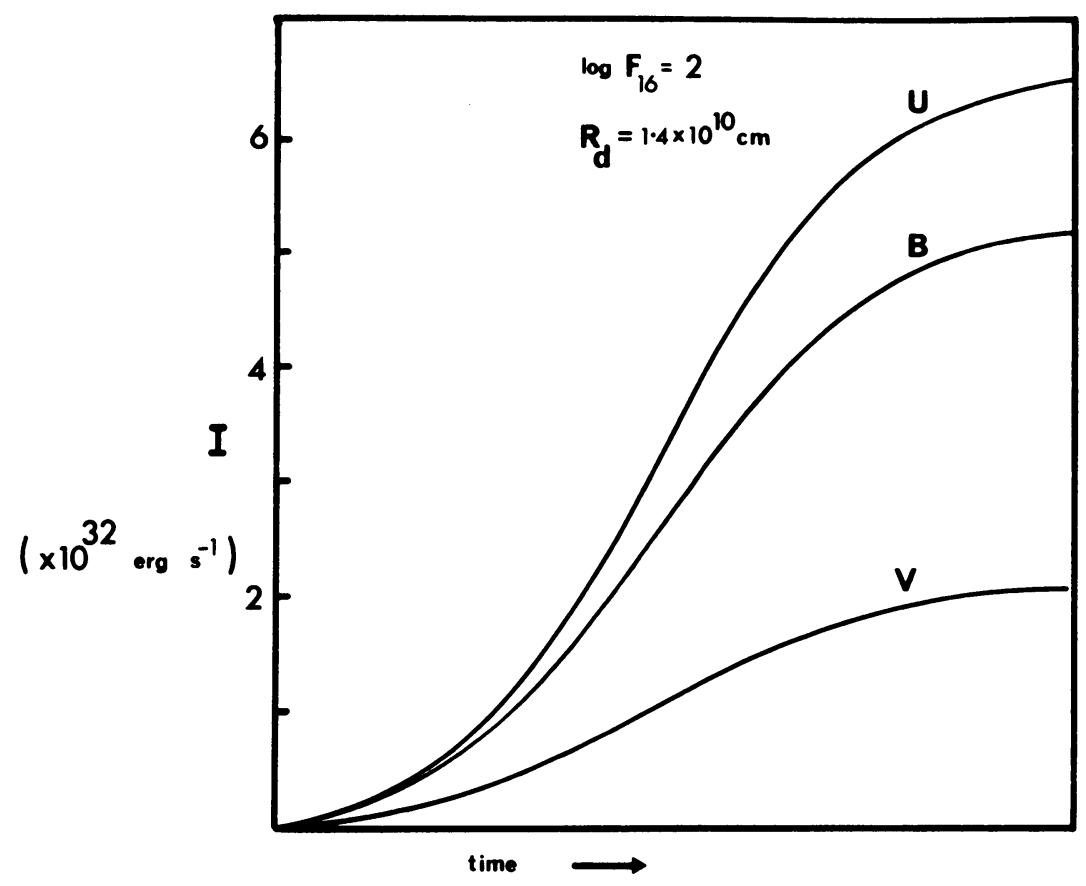

Fig. 8. Eclipse of a steady disc at $F_{16}=2$ (high accretion rates). A broad, $V$-shaped eclipse develops as the mean temperature of the disc increases.

the temperature, and therefore to the mass transfer rate. This is contrary to the optical situation where, at least at outburst, one is observing in the Rayleigh-Jeans region of the spectrum, and the optical flux is a relatively insensitive guide to the mass transfer rate. Furthermore the deduced soft $X$-ray flux will be very sensitive to the structure of the inner disc region and boundary layer (or to $\beta$ ). In Figure 9 the soft X-ray flux in the range 0.2 to $0.28 \mathrm{keV}$ is given for a typical dwarf nova disc for differing values of $\beta$. An indication of the reported sensitivity of present satellite detectors (in this case the ANS satellite) is shown. Clearly for mass transfer rates which produce reasonable optical outbursts $\left(10^{18}-10^{19} \mathrm{gm} \mathrm{s}^{-1}\right)$, the soft $\mathrm{X}$-ray flux would only be marginally detectable using present detectors on sources at $100 \mathrm{pc}$ with $\sin i=1$. The positive soft X-ray measurement of Rappaport et al. (1974) and the negative results of Henry et al. (1975) are thus not necessarily contradictory, or a serious problem for this model. A possibly serious problem to detecting soft X-rays from these sources is the importance of local absorption. Disc accretion leads necessarily to some mass loss, in order to carry away excess angular momentum from the disc. Indeed Robinson (1973) deduced mass loss rates of $\sim 10^{17} \mathrm{gm}$ $\mathrm{s}^{-1}$ from the strength of undisplaced emission lines in Z Cam. Such high mass loss rates could lead to large local absorption of any soft X-ray flux.

In this section we have shown that the simplest models of accretion disc structure give a reasonable account of the overall properties of dwarf novae outbursts. The luminosities, luminosity changes, and eclipse behaviour are natural consequences of the simplest accretion models. Serious problems concerning the time-dependent behaviour of the disc, 


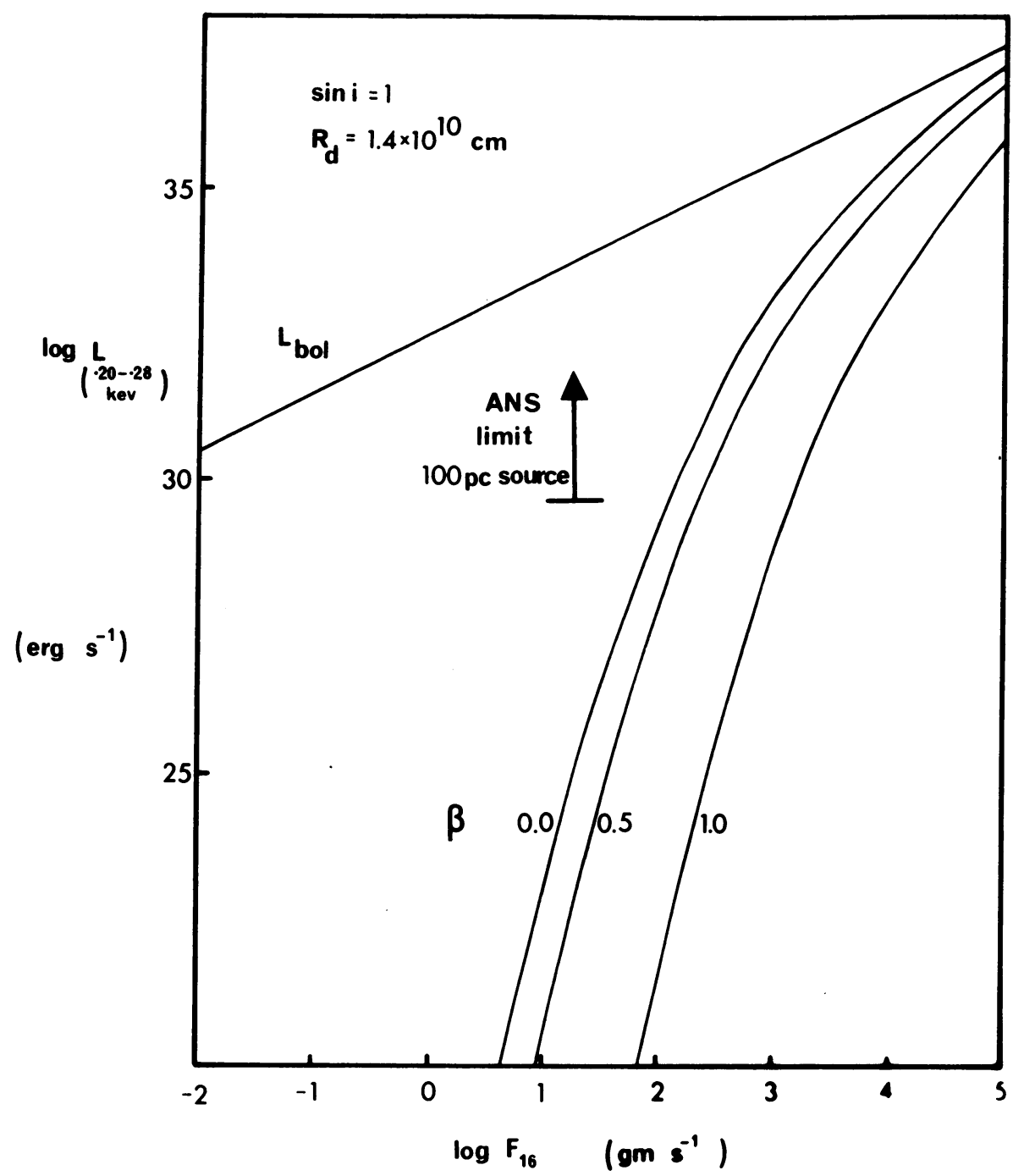

Fig. 9. Soft X-ray $(0.20-0.28 \mathrm{keV})$ luminosity of a steady accretion disc at different accretion rates. An indication of the sensitivity of a typical detector $\left(\gtrsim 10^{30} \mathrm{erg} \mathrm{s}^{-1}\right)$ on a $100 \mathrm{pc}$ source is shown.

the nature of viscosity in the disc, and the structure of the impact hot spot remain unanswered (though see Lynden-Bell and Pringle, 1974). However it is hoped that the case for a modulated accretion model of dwarf novae outbursts is now as clear as the case which has been put forward for a nuclear energy outburst model. In the previous section we summarized results that indicate that the modulation could be due to mass loss instabilities in the red component. However it is evident that Smak's third alternative an inherent disc instability model, would have many properties similar to those discussed here. 


\section{Conclusion}

Much work has been done on numerical studies of the nuclear burning processes that could, and should, eventually occur in these systems in which mass transfer onto a degenerate white dwarf is occurring. However we have stressed the way in which other physical processes can be important, some of which have been discussed in detail. Others, such as gravitational radiation by the binary, may also play an important role (Paczynski, 1967; Faulkner, 1971). These variables are complex, subtle and fascinating stellar systems. Their close similarity to classical novae, and general similarity to X-ray sources, makes them important members of the class of objects that we might call strongly interacting binaries. Within such objects a whole range of physical process must be occurring, and decoding which process is responsible for which particular observational feature is by no means a simple, self-evident matter. However in dwarf novae we have shown that the role of accretion as an energy source must be important. Studies of the red component stability and the accretion disc behaviour indicates that the many features of the outburst properties can be accounted for simply in terms of a quasi-periodic mass transfer model.

\section{Acknowledgement}

The modulated mass transfer/accretion model of dwarf novae discussed here is the result of a joint program with J. C. B. Papaloizou, J. E. Pringle and W. D. Evans. We thank J. Hazlehurst, M. Rees, B. Warner, A. Edwards, J. Ostriker, D. Moss, J. Whelan, A. Bolton and R. Carswell for discussions and contributions to this work.

\section{References}

Allen, C. W.: 1973, Astrophysical Quantities, Athlone Press.

Arp, H. C.: 1956, Astron. J. 61, 15.

Bath, G. T.: 1969, Astrophys. J. 158, 571.

Bath, G. T.: 1972, Astrophys. J. 173, 121.

Bath, G. T.: 1973, Nature Phys. Sci. 246, 84.

Bath, G. T.: 1975, Monthly Notices Roy. Astron. Soc. 171, 311.

Bath, G. T., Evans, W. D., Papaloizou, J. C. B., and Pringle, J. E.: 1974, Monthly Notices Roy. Astron. Soc. 169, 447.

Brickhill, A. J.: 1975, Monthly Notices Roy. Astron. Soc. 170, 405.

Crawford, J. A. and Kraft, R. P.: 1956, Astrophys. J. 123, 44.

Evans, W. D.: 1974, Unpublished Thesis, University of Oxford.

Faulkner, J.: 1971, Astrophys. J. Letters 170, L99.

Faulkner, J.: 1974, in R. J. Tayler (ed.), IAU Symp. 66, p. 155.

Finzi, A.: 1973, Astrophys. J. 183, 183.

Glasby, G. S.: 1970, The Dwarf Novae, Constable, London.

Giannone, P. and Weigert, A.: 1967, Z. Astrophys. 67, 420.

Henry, P., Cruddace, R., Lampton, M., Paresce, F., and Bowyer, S.: 1975, Astrophys. J. Letters 197, L117.

Joy, A. H.: 1954, Astrophys. J. 120, 377.

Joy, A. H.: 1956, Astrophys. J. 124, 317.

Kraft, R. P.: 1962, Astrophys. J. 135, 408.

Kraft, R. P.: 1963, Adv. Astron. Astrophys. 2, 43.

Kraft, R. P. and Luyten, W. J.: 1965, Astrophys. J. 142, 1041.

Krzeminski, W.: 1965, Astrophys. J. 142, 1051.

Lynden-Bell, D. and Pringle, J. E.: 1974, Monthly Notices Roy. Astron. Soc. 168, 603. 
Mumford, G. S.: 1969, Sky Telesc. 37, 288.

Novikov, I. and Thorne, K. S.: 1973, in C. de Witt and B. S. de Witt (eds.), Black Holes, Les Houches, Gordon and Breach, New York.

Osaki, Y.: 1974, Publ. Astron. Soc. Japan 26, 429.

Paczynski, B.: 1965a, Acta Astron. 15, 309.

Paczynski, B.: 1965b, Acta Astron. 15, 89.

Paczynski, B.: 1967, Acta Astron. 17, 287.

Paczynski, B.: 1971, Ann. Rev. Astron. Astrophys. 9, 183.

Papaloizou, J. C. B. and Bath, G. T.: 1975, Monthly Notices Roy. Astron. Soc. 172, 339.

Payne-Gaposhkin, C.: 1957, The Galactic Novae, North-Holland, Amsterdam.

Pringle, J. E. and Rees, M. J.: 1972, Astron. Astrophys 21, 1.

Rappaport, S., Cash, W., Doxley, R., MoClintock, J., and Moore, J.: 1974, Astrophys. J. Letters 187, L5.

Redkoborodyi, Y. N.: 1972, Astrofizika 8, 157.

Robinson, F. L.: 1973, Astrophys. J. 152, 245.

Shakura, N. J. and Sunyaev, R. A.: 1973, Astron. Astrophys. 24, 337.

Smak, J.: 1969, Acta Astronomica 19, 285.

Smak, J.: 1971a, Acta Astronomica 21, 15.

Smak, J.: 1971b, in IAU Colloq. 15, Bamberg, p. 248.

Starrfield, S.: 1976, this volume, p. 155.

Starrfield, S., Sparks, W. M., Truran, J. W., and Kutter, G. S.: 1972, Astrophys. J. 176, 169.

Starrfield, S., Sparks, W. M., and Truran, J. W.: 1974, Astrophys. J. Suppl. (in press).

Strand, K. A.: 1948, Astrophys. J. 107, 106.

Taam, R. E. and Faulkner, J.: 1975, Astrophys. J. 198, 435.

Terrell, N. J.: 1972, Astrophys. J. Letters 174, L35.

Walker, M. F.: 1954, Publ. Astron. Soc. Pacific 66, 230.

Walker, M. F. and Chincarini, G.: 1968, Astrophys. J. 154, 157.

Warner, B.: 1973, Monthly Notices Roy. Astron. Soc. 163, 25P.

Warner, B.: 1974a, Monthly Notices Astron. Soc. South Africa 33, 21.

Warner, B.: 1974b, Monthly Notices Roy. Astron. Soc. 168, 235.

Warner, B. and Brickhill, A. J.: 1974, Monthly Notices Roy. Astron. Soc. 166, 673.

Warner, B. and Harwood, J. M.: 1973, Inf. Bull. Var. Stars, IAU Comm. 27, No. 756.

Warner, B. and Nather, R. E.: 1971, Monthly Notices Roy. Astron. Soc. 152, 219.

Warner, B. and Robinson, E. L.: 1972, Nature Phys. Sci. 239, 2.

\section{DISCUSSION}

Faulkner: The instability mechanism which Dr Bath discussed was earlier employed by him and his colleagues (Evans, Papaloizou and Pringle) in association with their model of brightening accretion discs for dwarf nova outbursts. In that work, the authors make every effort to raise the temperatures as much as possible, so that very variable mass transfer rates could be deduced from moderate changes in optical luminosities. Nevertheless, if one accepts their analysis, the observations do not seem to demand that the outburst follows an increased rate of mass transfer. In VW Hyi, Warner has discussed the constancy of the hump as the background rose by large factors; in Z Cha, the behaviour of the hump several days after outburst suggests that there is then a variable rate of mass transfer, which may be so, but hardly seems germane to the reasons for onset. A more complete discussion will follow in my paper $(\S 3)$.

Pringle: There is some confusion about 'hot spots' in dwarf novae systems. What is meant by 'hot spots' by an observer is, for example, the hump in the light curve of U Gem, that is the anisotropic part of the radiation emitted from where the stream strikes the accretion disc. If the radiation from that point is emitted in an isotropic manner, no hump would be seen in the light curve and an observer would deduce that the luminosity of the hot spot is zero. Therefore, without a full understanding of the non-steady fluid interaction and radiation processes occuring at the intersection of the stream and the disc, it is naive to assume a correlation between the mass transfer rate and the intensity of the observationally defined hot spot.

Shu: How meaningful is a stability analysis of a situation which is not in equilibrium? The question of the stability of the steady-stated flow would seem to be more relevant than the case actually treated.

Bath: The stellar envelopes which we construct are in complete dynamical and thermal equilibrium, with the possible exception of an acoustically driven wind outside the photosphere. in exactly 
the same sense as any conventional stellar model. We fully realise that it would be more correct to include such a wind self consistently in the structure, but this problem is as yet unresolved even for single stars. The point of the instability we are discussing is that it is intrinsic to the physical structure of the envelope, and is essentially. driven by the ionization zones that exist at some depth below the photosphere. We do not believe that any attempt to construct some sort of steady outflow model will remove the essential physical cause of this instability.

Paczynski: I fully agree with $\mathrm{Dr}$ Bath that a nuclear burning instability cannot explain the luminosities and time scales characteristic for dwarf novae. However, I do not believe his nonlinear code produces credible results. I do not understand how his boundary conditions (i.e. a cut off at the Roche surface) can be proper, and neglecting convection is too much of a simplification. I think that as far as theory or speculation is concerned we have at the moment two competing possibilities. According to one we have highly nonsteady mass transfer from the red star to the disc, as presented by Dr Bath. But we have also a second possibility, considered recently by Osaki. According to that the mass transfer rate is more or less steady, but matter accumulates at the outer rim of the disc when U Gem is at minimum, and due to an unknown instability is accreted through the disk onto a white dwarf at the time of eruption. I hope the observations will help us to decide which viewpoint is correct.

Webbink: The question being raised here seems to be, in part, whether mass loss from the red component is steady, or modulated by a Bath-type instability. I will be speaking on Friday morning of an evolutionary calculation which gives strong indication of such an instability. Whether or not such an instability is in fact responsible for dwarf nova outbursts, I feel certain that the instability does exist.

Paczynski: The Z Cam stars have long periods of steady light. There are also systems like UX UMa, in which we see some sort of a steady mass transfer and accretion. I think the proper theoretical approach is to construct models with a steady flow first, and then study their stability. What you do with your linear analysis is a study of a turn-on effect, and not the stability of mass transfer. I think the observations show that in many systems the mass transfer rate is steady, and in some it may be unstable, but it is never off. So turn-on effects have nothing to do with the observed systems.

Hazlehurst: You showed the red component just inside the critical surface when the instability occurs. Does this mean a finite push is needed to remove material, and is this consistent with a linear analysis?

Bath: Equilibrium Roche models were constructed with photospheres at least one scale height below the critical lobe. The linear analysis of these models shows that an infinitesimal perturbation will grow exponentially until limited by non-linear terms. The photosphere will necessarily be lifted to the zero gravity point in a classical dynamical instability as more than enough energy is available from recombination in the underlying layers. As sequences of initial equilibrium models with decreasing photospheric radius are considered, so the unstable region contracts, goes marginally stable along the line of centres, and eventually becomes stable. Thus there is a critical radius (which is less than the Roche lobe radius) at which instability first sets in.

Hall: Paczynski contrasted the Bath model, in which mass flow is unsteady, with the Osaki model, in which the mass flow is steady. I want to point out that mass flow in all Algol binaries is very unsteady. Although the Algols and the cataclysmic variables are not identical, they are clearly related in that the mass-losing star is convective and fills its Roche lobe. Thus this observational fact may be useful in indicating that we might anticipate unsteady mass flow in the cataclysmic variables.

Whelan: Is it possible that the two versions of the accretion theory are compatible in the sense that the mass flow modulation instability leads to a disc instability? 